Revue d'histoire de l'Amérique française

REVUE D.HISTOIRE DE L'AMÉRIQUE FRANÇAISE

\title{
Les Corte-Real et le Nouveau-Monde (suite et fin)
}

\section{Eduardo Brazão}

Volume 19, numéro 3, décembre 1965

URI : https://id.erudit.org/iderudit/302489ar

DOI : https://doi.org/10.7202/302489ar

Aller au sommaire du numéro

Éditeur(s)

Institut d'histoire de l'Amérique française

ISSN

0035-2357 (imprimé)

1492-1383 (numérique)

Découvrir la revue

Citer cet article

Brazão, E. (1965). Les Corte-Real et le Nouveau-Monde (suite et fin). Revue

d'histoire de l'Amérique française, 19(3), 335-349.

https://doi.org/10.7202/302489ar d'utilisation que vous pouvez consulter en ligne.

https://apropos.erudit.org/fr/usagers/politique-dutilisation/ 


\title{
LES CORTE-REAL ET LE NOUVEAU-MONDE * (suite et fin)
}

III

\author{
MIGUEL CORTE-REAL \\ A LA RECHERCHE DE SON FRERE
}

La lettre de dom Manuel, en date du 15 janvier 1502, qui ratifiait la concession de la terre faite par Gaspar Corte-Real à son frère Miguel, commence ainsi :

"A quantos esta nosa carta virem, fazemos saber, que Miguell Corte Real, fidallguo de nossa casa, e nosso porteiro moor, nos disse ora, que vendo elle como Gaspar Corte Real, seu irmaao, avia dias que partira desta cidade com tres navyos a descobrir terra nova, de qual ja tinha achada parte della, e como depois de passado tempo vieram dous dos ditos navyos aa dita cidade, averiam cinquo messes, e elle nam vinha, que elle o queria hyr buscar..."

("... Nous faisons savoir que Miguel Corte-Real, gentilhomme de notre maison et notre Grand Portier, nous a dit que son frère, Gaspar Corte-Real, était parti de cette ville pour aller découvrir la nouvelle terre dont il avait déjà trouvé une partie; et comme il s'était écoulé cinq mois depuis et que deux de ses bateaux sont revenus à Lisbonne mais non le sien, lui, Miguel, désire aller à sa recherche ...") ${ }^{1}$

Ce désir s'inspirait évidemment d'une amitié familiale, mais peut-être surtout des intérêts matériels en jeu.

Miguel Corte-Real, second fils de João Vaz, avait non seulement accompagné son frère Gaspar dans son dernier voyage, mais l'avait aidé également à le financer et, de fait, partageait

* Voir notre Revue, XIX : 3-52, 163-202. 
avec lui les découvertes; c'est ce que dom Manuel ratifiait dans sa lettre. Il devait, dans un des navires de l'expédition, suivre la même route que son frère, entrer, possiblement, dans les mêmes baies, débarquer aux mêmes endroits, voir les mêmes contrées et parler aux mêmes indigènes. Personne n'était mieux préparé que Miguel Corte-Real pour retrouver Gaspar, si la mer ne l'avait pas déjà englouti.

Il devait également faire le partage de ces terres où croissaient des bois excellents pour la construction des navires de notre flotte, qui augmentait toujours. On y trouvait aussi des fourrures soyeuses et rares d'animaux presque inconnus en Europe, tel le castor, qui tenaient les Portugais au chaud durant les rudes hivers de ce pays ${ }^{2}$. Ce n'étaient pas tellement les richesses fabuleuses de l'Orient que l'on recherchait à cet endroit, comme on le pensait alors, mais plutôt un passage vers un nouvel Eldorado. Tout ceci semble découler du passage suivant de la lettre de 1502 :

“... e que por quanto elle dito Miguell Corte Real tinha feito muyto gasto e despesa de sua fazenda no dito descobrimento, asy nos ditos navyos que ho dito seu irmaão per ella armou per a primeira vez que a dita terra achou, e asy desta segunda que ora foy como com elle..."

(“. . au sujet duquel ledit Miguel Corte-Real avait encouru de grandes dépenses pour cette découverte, de même que pour lesdits navires que son frère avait gréés la première fois qu'il avait trouvé ladite terre et aussi cette seconde fois où il était avec lui...").

Que ora foy como com elle a été interprété: tivesse ido com ele (il était avec lui). C'est aussi ce que pense Gustave Lanctot: “...Au printemps de 1502, le deuxième Corte-Real, Miguel, qui avait fait le voyage, l'année précédente..."3. Or, d'après les deux documents des 6 et 7 août, publiés par Kunstmann ${ }^{4}$ et par Harrisse ${ }^{5}$, et que l'on trouve dans les archives de la Torre do Tombo (Lisbonne) ${ }^{6}$, l'on sait qu'à cette date, Miguel Corte-Real réclamait d'un nommé Cristóvão Lopes, écuyer du 
roi de Portugal, des vivres, épuisés, après trois mois, pour l'équipage de sa caravelle. Il s'agissait vraisemblablement du navire qui avait quitté Lisbonne au début de mai 1501.

Le plus étonnant, dans tout ceci, c'est que le reçu de ces approvisionnements est daté de Malega, qu'on a interprété comme Málaga. Tout le reste se tient. En réalité, selon Damião de Góis, l'expédition de 1501 de Gaspar Corte-Real "partio de Lisboa ahos XV dias do mes de Maio" (partit de Lisbonne dans les quinze jours du mois de mai). C'est à peu près la date où Miguel prenait la mer.

A propos de Málaga, il semble singulier qu'un navire à voile, partant de l'estuaire du Tage avec l'intention de traverser l'Atlantique, aurait été poussé sur la Méditerranée, même avec des vents cycloniques, qui ne sont pas normaux dans ces parages à cette époque de l'année. Et de plus, que faisait à Málaga un écuyer du roi de Portugal ? Nous n'y voyons qu'une seule explication: ce serait une erreur du copiste. Malega ne peut qu'être le nom de quelque port d'une des neuf îles des Açores; il ne reste plus alors qu'à assembler les morceaux de ce casse-tête chinois. Il est certain que ni Damião de Góis, ni António Galvão ne font allusion au voyage de Miguel et de son frère Gaspar en 1501. Il n'est pas étonnant toutefois: les deux récits étaient très succincts. Pour reconstituer les voyages des deux frères, il nous faut relier le peu de renseignements que nous ont laissés ces chroniqueurs, avec la cartographie qui en est résultée.

Le vaisseau de Miguel, d'après les documents que nous avons mentionnés, comptait 80 hommes d'équipage; de 50 au début, le roi lui ordonna "que tomase mais trimta..." (d'en prendre trente autres). Cela ressemblerait davantage, comme le dit Harrisse ?, à un début de colonisation qu'à l'équipage nécessaire à la manœuvre d'une caravelle: "Le nombre d'hommes embarqués, et, ensuite, par l'ordre du roi, augmenté, nous porte à croire qu'ils ne composaient pas un équipage, mais un renfort, peut-être en vue d'une colonie projetée, que le roi Manuel aurait envoyé à Gaspar Corte Real sous la conduite de son frère." 
Trois mois plus tard, Miguel était de retour puisque, le 4 novembre 1501, le roi Manuel lui accordait une pension annuelle de 30,000 réis: "A quamtos esta nosa carta virem fazemos saber que avemdo nos respeito ao(s) muytos seruiços que miguell corte reall fidalguo de nosa casa e noso porteiro moor tem feitos a elrej dom joham meu primo (D. João II) que samta glorea aja e outresy a nos e aos que ao deamte delle esperamos receber e queremdolhe fazer graça et mercee teemos por bem e nos (praz) ..."8 (Nous faisons savoir qu'en hommage aux grands services que Miguel Corte-Real, gentilhomme de notre maison et notre Grand Portier, a rendus à sa majesté dom Juan, (Jean II) mon cousin, et aussi à nous, de même que pour les autres services que nous attendons encore de lui, nous lui faisons la grâce de lui concéder...)

Par hypothèse, et d'après la suite logique des événements, nous croyons à une erreur dans la nomenclature, ou dans la transcription du mot Malega. Miguel Corte-Real, comme tout nous l'indique, accompagnait son frère Gaspar. Pour obéir aux ordres du monarque, il amenait avec lui un renfort d'hommes, au cas où il deviendrait possible non seulement de prendre possession des terres découvertes, mais d'y fonder une colonie. Le destin vint cependant contrecarrer les plans de ces nobles navigateurs au sujet de la Terre Verte, nom que Gaspar CorteReal lui donna, à cause de sa flore abondante. Ce nom devint plus tard Terre de Corte-Real sur les cartes postérieures à l'automne de $1503^{\circ}$, et commença à s'appeler Terre-Neuve par la suite.

La lettre royale de 1502 abordait ensuite précisément la répartition des terres qui faisaient l'objet de ces voyages au nord de l'Atlantique:

“...pelo que o dito Gaspar Corte Reall, avemdo respeito a isso, lhe prometera de partir com elle da dita terra que asy descobrisse asy e na maneira que a elle tinhamos outorgada e dada por nosa doaçam, da quall coussa o dito Gaspar Corte Reall nos pedio amte de sua partida, que lhe mandassemos disso 
dar hũu nosso alvara, o quall lhe demos a seu requerimento, pelo quall nos prouve, que toda a terra que lhe elle asy desse e demarcasse fosse sua, asy como a elle de nós tinha, e em sua carta era contheudo; e ora o dito Miguell Corte Reall nos pedio que pera sua segurança o decrarassemos asy e outorgassemos per esta nosa carta, pello quall, de nosso moto proprio, çerta çiencia, livre vomtade, poder Reall e aussoluto nos praz, que de toda a terra firme ou Ilhas que ho dito Gaspar Corte Reall atee ora tem achadas, ou descobrir daquella parte, que elle denomear e demarcar ao dito Miguel Corte Reall por sua, lhe fazemos della doaçam e merçee, pera todo sempre, como do fecto por esta fazemos, asy e tam cumpridamente, e com aquellas clausuallas e comdiçõoes, direitos, jurdiçam, capitanyas e coussas outras comtheudas na doaçam dedito Gaspar Corte Reall.

Outrosy nos praz, avemdo nos isso mesmo respeito ao que dito he, e asy aos muytos serviços que temos recebidos, e ao diamte esperamos receber do dito Miguel Corte Reall, que seemdo casso que elle nom ache o dito sseu irmaão, ou sendo falecido, o que Deus nam mande, queremos e nos praz, que toda a terra firme e ilhas que elle per si novamente neste anno de quinhemtos e dous descobrir e achar, alem da que o dito seu irmaão tever achada, elle a aja pera sy, e lhe fazemos della doaçam e merçee, com aquellas jurdicõoes, direitos, capitanyas, clausullas, comdiçoões e coussas outras comtheudas e decraradas na dita doaçam do dito seu irmaõo, e por firmeza de todo lhe mandamos dar esta carta per nos asinada, e sellada do nosso sello pendente. Dada em Lixboa a $\mathrm{xb}$ dias de Janeiro. Gaspar Rodrigues a fez, anno do nosso Senhor Jhûu $X^{\circ}$ demill e b e dous - e daquellas terras ou ilhas que ho dito sseu irmaão asy tever achadas e descobertas nom lhe fazemos doaçam, ssoomente daquellas que lhe asy nomear como dito he."

(... par laquelle ledit Gaspar Corte-Real promet de partager avec lui (Miguel) ladite terre qu'il découvrirait, de la même façon que nous, par nos lettres, la lui donnons et transmettons à lui (Gaspar), et 
ledit Gaspar Corte-Real nous demande de lui donner nos lettres patentes de ladite concession avant son départ, lesquelles lettres nous lui remettons, à sa demande, et par lesquelles c'est notre bon plaisir que toute la terre qu'il donnerait ou recevrait de lui (Miguel) serait sienne, de la même manière que lui (Gaspar) la tient de nous, comme il est stipulé dans ses lettres patentes; et ledit Miguel Corte-Real nous a demandé, pour sa protection, de déclarer ceci et de donner la même chose par nos lettres par lesquelles, de notre propre désir, pleine connaissance, entière volonté et pouvoir royal absolu, il est de notre bon plaisir de lui accorder et remettre pour toujours toute partie de terre ferme ou îles découvertes ou trouvées dans cette région par ledit Gaspar Corte-Real, qu'il a désignée et réservée pour ledit Miguel Corte-Real, et nous faisons cette donation de la même manière et aussi complètement et avec ces clauses, conditions, droits, juridiction, attributs de capitainerie et autres titres qui sont établis dans la donation audit Gaspar Corte-Real.

C'est également notre bon plaisir, en rapport avec ce qui est déclaré, et aussi en tenant compte des services que nous avons recus et que nous espérons recevoir dans l'avenir dudit Miguel Corte-Real, que s'il ne trouvait pas son frère (Gaspar) ou que celuici soit mort, Dieu l'en protège, c'est notre désir et notre plaisir qu'il ait pour lui-même, et nous lui donnons et concédons toute la terre ferme et les îles qu'il peut découvrir ou trouver de nouveau en cette année 1502, en sus de celles que son frère a trouvées, avec toute juridiction, droits, capitainerie, clauses, conditions et autres titres contenus et stipulés dans lesdites lettres patentes à son dit frère; en foi de quoi nous avons donné ces lettres, signées et scellées de notre grand sceau, pour lui être remises. Donné à Lisbonne, le 15 janvier. Gaspar Rodrigues a fait ceci en l'année de Notre Seigneur Jésus-Christ 1502. Et nous ne lui donnons pas ces terres et îles que son frère a trouvées et découvertes, mais seulement celles que nous avons déjà nommées, tel que déclaré précédemment.) 
Miguel Corte-Real, comme nous le savons déjà, était le deuxième fils du truculent João Vaz. On croit qu'il est né avant 1450, à Tavira ou à Lisbonne. Haut personnage de la cour, où il accéda à la fonction de Grand Portier du Roi Fortuné (D. Manuel). Contrairement à Gaspar, il ne semble pas qu'il ait vécu à l'île Terceira. Il fut surtout un palatin. Il épousa, avant 1490, une fille de Garcia de Castro: Isabelle, qui lui donna deux enfants: Catherine, qui épousa Diogo de Melo da Silva; et Joana, la femme de Lionel de Sousa, seigneur d'Ericeira ${ }^{10}$. Cette Isabelle Corte-Real vécut très longtemps puisque le 10 octobre 1576 elle signait un acte de vente ${ }^{11}$.

Après avoir reçu la lettre patente ratifiant le partage des terres découvertes et la concession de celles qu'il découvrirait, dont nous avons déjà parlé, Miguel partit, le 10 mai 1502, à la recherche de son frère, avec deux caravelles, d'après Damião de Góis, ou trois, comme le prétend Antonio Galvão ${ }^{12}$.

Malheureusement, comme dans le cas du voyage de Gaspar, nous n'avons pu trouver, jusqu'aujourd'hui, de récits provenant de témoins qui auraient entendu les marins des caravelles raconter leur voyage en rentrant à Lisbonne. Tout ce que nous pouvons supposer se trouve dans Galvão, qui relata les faits longtemps après, et surtout dans les cartes de Cantino, Canerio, Hamy King et Kunstmann II, citées par Harrisse ${ }^{13}$, sans doute d'après des originaux portugais. Il est possible que ces originaux soient l'esquisse, tracée par les navigateurs eux-mêmes, de leurs voyages à la recherche des deux frères disparus. Nous avons en plus les cartes mêmes de nos cartographes de cette époque.

Galvão, de son côté, raconte: “... Fazendo outra vez este caminho, se perdeo o navio em que elle hia (Gaspar), \& o outro tornou a Portugal. Pola qual causa seu yrmão Miguel corte real foy em sua busca cõ tres navios armados ã sua custa. Chegados àquella costa, como virão muytas bocas de rios \& abras, entrou cada hum pela sua com regimento que se ajũtassẽ todos ate vinte dias do mes Dagosto: os dous nauios assi o fizerao. $E$ vendo que não vinha Miguel corte real ao prazo, nem despois algum tempo, se tornarã a este Reyno, sem nũca mais delle se saber noua, nem ficar outra memoria, se não chamarse esta 
terra dos Corte Reaes ainda agora." (Parcourant encore une fois cette route, la caravelle qui transportait Gaspar s'est perdue et l'autre retourna au Portugal. Pour cette raison, son frère Miguel partit à sa recherche avec trois navires gréés à ses frais, Arrivés à cette côte, ils aperçurent plusieurs embouchures de fleuves et y pénétrèrent; en se séparant, ils convinrent de se rencontrer à cet endroit dans vingt jours pour retourner au Portugal. Deux navires seulement se rencontrèrent dans le délai fixé et retournèrent au Portugal. Celui de Miguel n'y était pas et on n'en entendit plus parler. L'unique souvenir qui en reste est que la terre qu'ils avaient découverte commença à être connue sous le nom de terre de Corte Real jusqu'à maintenant.) ${ }^{14}$

Sur ces données, et il n'en existe pas d'autres jusqu'à maintenant, sauf la cartographie contemporaine, Biggar écrit ${ }^{15}$ :

"Arrivé à Terre-Neuve, ordre fut donné à chaque vaisseau de visiter chacun sa partie de la côte et de se rassembler à SaintJean le 20 août. Il semble que l'un des vaisseaux visita la région située au delà de la baie Placentia, endroit où Gaspar s'était séparé de ceux qui l'avaient accompagné. Chapeau Rouge, sorte de colline conique que forme l'extrémité de la baie à l'ouest, reçut apparemment le nom de cap Pilot (C. do Piloto sur les cartes de Viegas et de Riccardiana), tandis que Langlade fut appelée île Green ( $I$. Verde, sur les cartes de Viegas et de Riccardiana) pour faire contraster celle-ci avec la terre ferme stérile située en face. Le cap La Hune, point de reconnaissance important situé à l'ouest, fut appelé cap Shallop (C. do Batell sur la carte de Viegas. La $b$. do Batell sur l'atlas de Riccardiana serait donc la baie Hermitage), peut-être parce que l'une des chaloupes de Gaspar fut trouvée à cet endroit. Près de GrandBruit les sauvages ont apparemment cherché à faire des échanges et par suite, les Portugais auraient appelé ce promontoire Peddler's cape ( $C$. do Mazcatos sur l'atlas de Riccardiana; $c$. do Mazcato sur la carte de Viegas).

Après avoir doublé le cap Ray qui fut appelé avec raison cap Bend (C. da Volta sur les cartes de Viegas et de Riccardiana) 
le vaisseau s'avança à une distance considérable le long de la côte ouest de Terre-Neuve. La baie et la rivière Saint-Georges qui furent trouvées plus agréables que toute autre partie de la côte sud, reçurent le nom de rivière Pretty (Rio Fremoso sur les cartes précédentes). Cette baie est séparée de la baie Portau-Port par un isthme étroit dont la largeur en certains endroits est moins de cent verges, ce qui valut à la baie de Port-au-Port le nom de baie Athwart (Rio da Travesa sur les cartes de Viegas et de Riccardiana). La baie des Islands dans laquelle se jette la plus grande rivière de Terreneuve reçut le nom de rivière Large (Rio Comprido sur l'atlas de Riccardiana) tandis que la baie Bonne dont la largeur est de quatre milles seulement fut appelée baie Small (B. Pequena sur l'atlas de Riccardiana). La côte élevée jusque-là se transforme à cet endroit en place inclinée désignée sous le nom de "plage unie" (Costa chã sur les cartes de Viegas et de Riccardiana). Des sauvages ayant été rencontrés à la baie Saint-Paul, celle-ci reçut le nom de rivière des Natives (Rio das Poblas sur la carte de Viegas et $B$. de Solhas sur l'atlas de Riccardiana). Ce vaisseau ne semble pas être allé plus loin que Cow-Head qui fut appelé cap du Gulf ( $C$. do Golfram sur l'atlas de Riccardiana) car c'est de là qu'il partit pour le rendez-vous à Saint-Jean.

A cet endroit il rencontra l'un des autres vaisseaux tel qu'il avait convenu, mais ni l'un ni l'autre n'avait découvert d'indice de Gaspar Corte Real et par surcroît de malheur, le vaisseau de Michael manquait. Après avoir attendu en vain pendant quelque temps, les deux vaisseaux prirent seuls la route du Portugal."

Mais Biggar aurait pu aller plus loin, car un homme comme lui qui a scruté le travail consciencieux et détaillé d'Henry Harrisse sur la Découverte et évolution cartographique de Terre Neuve, publié en 1900, peut avoir une idée précise de la richesse de la toponymie portugaise du Labrador et de Terre-Neuve. Même quand quelques-uns des noms de cette région, sur tant de cartes, ne semblent pas écrits dans notre langue, on finit par croire que le cartographe s'est trompé en copiant les originaux de cette nomenclature, écrits dans une langue étrangère. 
Avec toutes ces preuves, qu'on a injustement cherché à effacer, peut-il subsister un doute quant à la découverte de Terre-Neuve par ces braves navigateurs açoréens ?

Ici devrait se terminer le voyage de Miguel Corte-Real. Mais la légende, que nous ne pouvons ignorer, le projette bien plus avant.

Sur la rive droite du fleuve Taunton s'élève la ville de Dighton, dans le minuscule État de Massachusetts, tout près de nous. A un demi-mille de là se dressait un rocher appelé, sans autre indication, Dighton Rock, aujourd'hui dans un jardin publique - Dighton State Park. Malgré les marées, un grand nombre d'inscriptions y sont restées gravées, faites peut-être par quelque passant amoureux qui y aurait griffonné ses sentiments. Pour nous, latins et méridionaux, ce genre de souvenirs et de confidences sont très communs.

En 1920, l'Américain Edmond Delabarre ${ }^{16}$ crut découvrir dans cet enchevêtrement de signes, à côté de l'emblème de notre drapeau: MIGVEL CORTE REAL V DEI hic DVX. Et aussi : A.D. 1511. Ce qui pourrait se lire de cette façon: Miguel CorteReal hic Dux Ind(orum), A(nno) D(omini) 1511, et se traduirait ainsi: Miguel Corte-Real aqui chefe dos Indios no Ano de 1511. (Miguel Corte-Real, ici, le chef des Indiens, en l'an 1511.)

Or, Miguel partit de Lisbonne en mai 1502. Les trois vaisseaux se séparèrent pour chercher l'île, et avec l'espoir de trouver quelque trace de Gaspar. Ils devaient se rencontrer le 20 août pour le voyage de retour ou pour tenter d'autres recherches si les premières échouaient. Or, de 1502 à 1511, il s'est écoulé neuf années. Il n'est pas possible qu'il restât ancré devant la côte durant tant d'années ! Et pourquoi aurait-il attendu si longtemps pour graver son nom dans la pierre?

La thèse de Delabarre s'appuie historiquement sur la tradition exposée par John Danforth, et beaucoup plus tard par Edward A. Kendall, au sujet du passage sur ce fleuve d'un navire ou "oiseau de bois", d'où sont descendus des hommes blancs qui 
se sont battus contre les Indiens aguerris de cette région. Les Blancs auraient été massacrés, et Miguel Corte-Real, réussissant à s'échapper, devint, on ne sait par quel miracle, chef de cette tribu !...

Que les Portugais soient descendus là, nous en sommes convaincus, mais nous ne pouvons croire à cette histoire de Delabarre, tout intéressante qu'elle soit, qui, nous semble-t-il, depuis l'époque où elle a commencé à circuler, a attiré beaucoup de touristes et de curieux aux rives du Taunton et au jardin de Dighton.

S'il s'agissait de Gaspar, cela pourrait être plus plausible puisque celui-ci ordonna le retour à Lisbonne de deux de ses vaisseaux pour continuer son exploration vers le sud. Il désirait constater la relation entre cette côte découverte par les Portugais et les îles où Christophe Colomb avait abordé, peu avant. ${ }^{17}$ Cantino ${ }^{18}$ disait: "L'altro compagno (Gaspar) ha deliberato andar tanto per quella costa che vole intendere se quella è insula o pur terra ferma". Et Pietro Pasqualigo ${ }^{19}$ : Qual terra etiam credeno conjungerse con le Andilie, che furono discoperte per li Reali di Epagna, et con la terra dei papaga, noviter trovata per la nave di questo se che andorono in Calicut. El creder questo se moveno, per che havendo corsa la costa de dita terra per spazio de 600 et piu miglia, non hanno trovato fin alguno; poi, perche diceno haver trovate molte fiumare grosissime che li meteno in mare." Pourtant, avant Verrazane, les Portugais en étaient déjà venus à la conclusion de l'existence du continent américain.

La disparition de gentilhommes de si haute lignée et si attachés à la couronne portugaise causa une impression profonde sur le monarque régnant. Damião de Góis écrit: "Ha perda destes dous irmãos sentio el Rei muito, pela criaçam q. nelles fezera, pelo que mouido de seu real, \& piadoso moto, no anno seguinte de M.D. III mandou duas naos armadas à sua custa, buscalos, mas nem de hum, nẽ de outro se põde nunqua saber onde, nem quomo se perderam, pelo q. se pos. á quella prouinçia 
da terra verde, onde se cre que sestes dous irmãos perderam, ha terra dos corte Reaes." ("Le roi fut très affecté par la perte de ces deux frères parce qu'il les connaissait depuis longtemps et les aimait beaucoup. Mû par ce sentiment, il envoya, l'année suivante (1503) deux caravelles, payées par lui, à leur recherche. Mais ni l'un ni l'autre ne fut retrouvé. Pour ce motif, on a donné à la Terre Verte, où on croit ces deux hommes disparus, le nom de Terre de Corte-Real".)

On ne sait rien de ce voyage mais, d'après Biggar ${ }^{20}$, les vaisseaux de dom Manuel ont dû explorer la même région, aperçue en 1501 et en 1502 .

Lorsque les deux frères, Gaspar et Miguel, furent considérés perdus, leurs droits sur les terres découvertes et dont ils avaient pris possession, passaient à Vasco Anes, leur aîné ${ }^{21}$ : "veador dâ casâ del Reí, do seu Conselho capitam e governador das ilhas de Sam George, \& terceira, \& alcaide môr da çidade de Tauira" ("... de la maison du roi, capitaine de son conseil, gouverneur des îles Saint-Georges et Terceira et grand alcade de la cité de Tauira"), comme l'écrit le chroniqueur de dom Manuel, qui ajoutait aux titres officiels, les mérites personnels: "muito bom caualleiro, bom Christão, homẽ de singular exêmplo de vida, $\&$ de muitas esmollas publicas, et secretas." ("très bon cavalier, bon chrétien, homme d'un genre de vie exceptionnel, aux nombreuses aumônes publiques et privées.")

Celui-ci désirait également partir, avec des caravelles gréées à ses frais, car il se refusait à croire à la mort de ses frères. Mais le roi ne le lui permit pas; cette noble maison portugaise avait déjà versé suffisamment de sang. Nous avions besoin d'hommes valeureux pour nos découvertes, que devait suivre l'œuvre de colonisation, c'est-à-dire l'éducation des peuplades primitives rencontrées en Afrique et en Amérique.

En 1580, Philippe II d'Espagne, après la mort tragique du jeune roi de Portugal, dom Sébastien, tué dans une bataille au nord de l'Afrique, devint prétendant au trône portugais, à cause 
de ses liens de parenté avec notre famille royale, et l'absence de successeur direct au monarque défunt. Un tel droit était contestable. Mais la meilleure de nos forces militaires portugaises fut écrasée dans cette bataille où nous avions placé tant d'espoirs. Ainsi commença l'union personnelle entre le Portugal et l'Espagne déjà unifiée. Cette situation politique dura 60 ans.

Mais la petite île Terceira, berceau de quelques illustres navigateurs portugais, réagit violemment. Il devint nécessaire à la puissante armada de $\mathrm{D}$. Alvaro de Bazan, marquis de Santa Cruz, de l'écraser pour la vaincre. Ceci se passait en $1583^{22}$. Le seigneur de Bazan, bien qu'il fût Grand d'Espagne, en foulant ce sol, aurait dû se découvrir et s'incliner devant ce peuple héroïque.

Coïncidence du Destin : au cours de cette même année, les Portugais de Saint-Jean recevaient solennellement Sir Humphrey Gilbert qui, au nom d'Elisabeth $\mathrm{I}^{\mathrm{re}}$, venait prendre possession de ce que le Portugal, légitimement, considérait déjà comme sien. Plus tard, ce furent les Pays-Bas qui s'emparèrent d'une partie de son Brésil et de son Angola, et enfin les Anglais qui occupèrent aussi des parties de son Orient. Le Portugal déclinait, mais il regardait son passé avec fierté: non seulement avait-il été le découvreur, mais aussi et surtout le créateur de nouvelles nations où le mot démocratie, que nous entendons aujourd'hui dans des sens variés et souvent contradictoires, avait reçu sa pleine acception chrétienne.

\section{EdUARdo BRAzao}

1 H. P. Biggar, op. cit., 67; Harrisse, op. cit. doc. $\mathrm{XX}$ de l'appendice, 214 et suiv., Torre do Tombo, liv. IV de D. Manuel, fol. 3v., pub. par Kunstmann, Die Entdeckung Amerikas, Nach den ältesten Quellen geschichtligh dargestellt (München, 1859), 93, note 120 .

2 Voir l'excellent ouvrage de l'économiste canadien Harold A. Innis, The fur trade in Canada, an introduction to Canadian Economic History (Nouvelle édition, "University of Toronto Press", 1962), l'introduction "The Beaver", 3 et suiv. et le premier paragraphe du deuxième chapitre: "Beginning of the Fur Trade on the Atlantic Coast" (1497-1600), 9 et suiv. Le castor a été aussi beaucoup utilisé en Europe pour la confection de chapeaux d'hommes.

${ }^{3}$ Gustave Lanctot, Histoire du Canada, I, des origines au régime royal

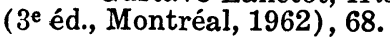


4 Op. cit., 93, note 119.

5 Op. cit., doc. $\mathrm{n}^{\text {os }} \mathrm{XIV}$ et XV de l'Appendice, 201 et suivantes: 1) 6 août 1501

"Senhor xpãm lopez quando armey em lyxboa eu tomei mãtimento pera tres meses. S. pera cinquventa homes e depois mandou el rey noso senhor que tomase mais trimta homes pera os quaes nõ pude tomar mais mãtimento por nõ caber no nauyo. aguora ha acerqua de tres (dias) que se gastou de guisa que aribei aqui por migoa dele e por ponete que venta que me nõ deyxa ir peçovos por merce que me mãdes dar duas pipas de vinho e hũ boy ou $\mathrm{xb}$ ou $\mathrm{xx}$ aroubas de carne e isto vos peço da parte del rey e peço merce a ffernã dalcaçova que ve a nissycidade minha que vos dedisto huma certidã. fecto aos seis dias dagosto de quinhentos e hum"

2) 7 août 1501

"Eu miguell corte reall diguo $q$ he verdale $q$ reçeby de xpãm lopez escudeiro del rej nosso (senhor) duas pipas de vinho e $\mathrm{xx}$ arobas de carne as quaes duas pipas de vynho e vynte arobas de carne asy recebo para mãtimento de oytenta homes, aos quaes faleceo ho mãtimento $q$ pera eles trazia aos sete dias do mes dagosto e porq assy he verdade $\mathrm{q}$ o dito mãtimento dele recebeo lhe dey este fecto aos sete dias do dito mes dagosto da era de quinhentos e hum - em malega

- Corpo Cronológico, Partie II, liasse 4, n 141.

7 Op. cit., 165.

8 Torre do Tombo, liv. XLIV de D. Manuel, fol. 95. Harrisse, op. cit., doc. $\mathrm{n}^{\circ} \mathrm{XIX}$ de l'Appendice, 213 ss.

9 Voir Henry Harrisse, Découverte et évolution cartographique de Terre Neuve et des pays circonvoisins, 1497-1501-176.9, essais de géographie historique et documentaire (Paris, Londres, 1900), 50.

10 Torre do Tombo, liv. V des doaçõas de D. Manuel, fol. 22; Harrisse, Les Corte Real ..., 169; encore dans le Torre do Tombo, Corpo Chronologico, part. II, liasse 22 , doc. $\mathrm{n}^{\circ} 37$; Livro das Ilhas, fol. 163v., Harrisse, Décou verte ..., doc. XXIV et XXV, Appendice, 222 ss.

11 Inventario dos Livros a mais Papeis que existem no Archivo desta Real Caza do Hospital do Espirito Santo, Tavira, $1^{\mathrm{er}}$ octobre 1825: "A fl. 29 está uma escritura de venda de um foro de $2 \$ 000$ reis pagos no dia de Santa Iria, feito por Domingos Vaz e sua Mulher a D. Izabel, mulher que foi de Miguel Corte Real, imposto numa sua fazenda do termo de Cacela, constituida por figueiras, oliveiras e poço de água na Serra, partindo com propriedades de Martim Afonso, Damião Martins e outros na Corte do Gago. 1576 Outubro 10" - Descobrimentos portugueses - o Algarve e os Descobrimentos par Alberto Iria, vol. II, tome I (Lisbonne 1956), 39.

12 Voir ces chroniqueurs - Damião de Gois, Crónica cit., 146 et suiv.; Galvão, dans la dernière édition de son œuvre, 97; Harrisse, Découverte...., 48.

13 Op. cit., 49.

14 Antonio Galvão, op. cit., dans sa dernière réédition citée à la page 97.

15 Op. cit., XVIII de l'Introduction.

16 E. Delabarre, The earliest and most puzzling of New England antiquities, dans "Old-Time New Englans", (octobre 1923) ; voir également Francisco Fernandes Lopes, Os irmãos Corte-Real, (Lisbonne, 1957); Gilberto S. Marques, Pedra de Dighton (New York, 1930); J. M. Cordeiro de Sousa, A inscrição Portuguesa na Pedra de Dighton, dans "Petrus Nonius", 
vol. II, fasc. 2, (Lisbonne, 1939) ; Damião Peres, História dos Descobrimentos Portugueses, (Porto, 1943), 356 ss.

17 Biggar, op. cit., XVII de l'Introduction: "Le fut vers cette époque que dans l'un des ports sur la côte ouest de la baie Placentia, Corte Real se sépara de deux de ses vaisseaux qui prirent la route de Lisbonne. Il voulait continuer son exploration vers le sud afin de s'assurer des communications entre ce grand continent et les îles que Christophe Colomb avait découvertes près de l'équateur".

18 Dans Biggar, op. cit., doc. XXII, 62.

19 Dans Biggar, op. cet., doc. XXIII, 65 et 66.

20 op. cit., $\mathrm{XX}$ de l'Introduction.

21 Lettres patentes du 17 septembre 1506, dans Biggar, op. cit., 92.

22 Henrique Braz, op. cit., 11; M. A. Buchanan, Early Canadian History, "Royal Society of Canada", vol. XLII, série III, (mai 1948), 43. 